\title{
An international perspective on outreach
}

Animal research is a complicated and delicate topic. Although its benefits, through enhanced biomedical knowledge and clinical treatments, can be felt throughout the world, some people believe that the use of animals for scientific and medical discoveries is simply unacceptable. Even among those that do support animal research, some might still consider it to be merely tolerable and a 'necessary evil'. Adding more complexity to the issue are the strong and historical international differences in views towards animal research.

Compared with our European counterparts, US biomedical researchers have largely enjoyed strong support, both public and federal, for their work, owing to surges in popular interest in science during the cold-war era, and the resulting large increases in funding across government agencies, including the NIH. The great strides in US scientific progress, regardless of discipline, have always been a source of pride. This isn't to say that researchers in the United States have never felt the wrath of animal rights activists, but arguably, as a whole, it has been less intense than what animal researchers in Europe and elsewhere have endured.

The good news-for Europe-is that openness and support for biomedical research using animals has significantly improved over the past decade. The efforts of several advocacy groups, including Pro-Test, the European Animal Research Association (EARA) and Understanding Animal Research (UAR), have been critical to this improvement. An additional crucial component to the changing tide in views was the establishment of the National Centre for the Replacement, Refinement \& Reduction of Animals in Research (NC3Rs), which provides funding and support specifically for lab animal science and improved welfare-imagine a branch of the NIH dedicated to funding animal welfare science! This support system has created a culture more open and active in its efforts to engage the public about the benefits of biomedical research using animals, the dedicated work that takes place to ensure ethical and humane treatment, and the continual push for improved welfare through science.

In this issue of Lab Animal there are two articles describing important topics and updates in animal research advocacy across Europe. In a News Feature, Wendy Jarrett, Chief Executive of UAR, writes about the recently launched Concordat on Openness in Animal Research, which is a signed commitment by institutions to enhance their openness and public communication about their use of animals in research. The Concordat sets specific guidelines for this commitment and tracks progress through annual reports. Kirk Leech, Executive Director of EARA, writes in this month's Outreach column about World Day for Laboratory Animals. Originally developed by anti-research groups in Europe to protest and propagate falsehoods about biomedical research involving animals, World Day for Laboratory Animals is now being used as an opportunity for the biomedical community to reach out to the public about the importance of research with animals. Kirk's article focuses specifically on EARA's efforts to get ahead of the curve in Belgium, and to help move institutions away from defensive silence toward active engagement with the media and public on World Day for Laboratory Animals.

In addition to these two articles, readers might have noticed a recent trend in Lab Animal's international reporting on research advocacy, as exemplified in Kirk Leech's recent article (Lab Anim. (NY) 45, 189; 2016). This is not by accident. While Europe has made significant gains in public support for animal research-some setbacks notwithstanding - the United States is struggling to maintain its high approval levels of decades past. Part of this is owing to the inevitable influence of time; many of us are now at least one generation removed from diseases like polio, blissfully unaware of the vital role that animal research played, and still plays, in developing life-saving vaccines and treatments. Much of US society can now take for granted 
long life expectancies and extraordinary medical treatments due in large part to the gains made from biomedical research with animals. Other forces, however, are also at play.

Today it is easy to spread lies quickly through social media, and a dangerous mixture of misinformation from anti-research groups and quiescence from the biomedical community is threatening public support for the very research that improves the quality of life for so many. Adding to this threat are the highly publicized failures in reproducibility and translatability of animal disease models. Much of the support for biomedical research rests on the assumption that the use of animals is justified by significant decreases in human suffering through improved science and clinical treatments.

The United States should learn from the valuable lessons of European biomedical research. If the response to threats is more silence from the research community, we are unlikely to succeed in changing negative trends. If Congress and the NIH simply produce more regulatory burden, without acknowledging - and properly funding the important link between animal welfare and improved disease models and reproducibility, the negative public outlook on biomedical research is likely to continue. 